Pacific Journal of Mathematics

ON MEANS OF DISTANCES ON THE SURFACE OF A SPHERE 


\section{ON MEANS OF DISTANCES ON THE SURFACE OF A SPHERE (LOWER BOUNDS)}

\section{Gerold WAGNeR}

Given $N$ points $x_{1}, x_{2}, \ldots, x_{N}$ on a unit sphere $S$ in Euclidean $d$ space $(d \geq 3)$, we investigate the $\alpha$-sum $\sum\left|x-x_{J}\right|^{\alpha}, \alpha>1-d$, of their distances from a variable point $x$ on $S$. We obtain an essentially best possible lower bound for the $L^{1}$-norm of its deviation from the mean value. As an application, we prove similar bounds for the $\alpha$-sums $\sum\left|x_{j}-x_{k}\right|^{\alpha}$ of mutual distances.

Introduction. Let $S=S^{d-1}$ be the surface of the unit (hyper)sphere in $d$-dimensional Euclidean space $(d \geq 3)$. Denote by $|x-y|$ the Euclidean distance between two points $x$ and $y$ on $S^{d-1}$. Let $\omega_{N}=$ $\left(x_{1}, x_{2}, \ldots, x_{N}\right)$ be a fixed set of $N$ points on $S$, and let $x \in S$ be a variable point. With each value of a parameter $\alpha(1-d<\alpha<\infty)$ we associate a distance function $U_{\alpha}\left(x, \omega_{N}\right)$ on $S^{d-1}$, which we define as follows:

$$
\begin{aligned}
& U_{\alpha}\left(x, \omega_{N}\right)=\sum_{j=1}^{N}\left|x-x_{j}\right|^{\alpha}-N \cdot m(\alpha, d) \quad(\alpha \neq 0), \\
& U_{0}\left(x, \omega_{N}\right)=\sum_{j=1}^{N} \log \left|x-x_{j}\right|-N \cdot m(0, d) \quad(\alpha=0) .
\end{aligned}
$$

Here $m(\alpha, d)$ is the mean value of $\left|x-x_{j}\right|^{\alpha}$ on $S$, which means

$$
\begin{aligned}
& m(\alpha, d)=\frac{1}{\sigma(S)} \int_{S}\left|x-x_{j}\right|^{\alpha} d \sigma(x) \quad(\alpha \neq 0), \\
& m(0, d)=\frac{1}{\sigma(S)} \int_{S} \log \left|x-x_{j}\right| d \sigma(x) \quad(\alpha=0),
\end{aligned}
$$

where $\sigma$ is the $(d-1)$-dimensional area measure on $S$.

We give two interpretations of the functions $U_{\alpha}$. First, the sums $\sum\left|x-x_{j}\right|^{\alpha}$ are related to the classical $\alpha$-means $\left(\frac{1}{N} \sum\left|x-x_{j}\right|^{\alpha}\right)$ of distances from the point $x$ to the points of $\omega_{N}$, which contain as special cases the arithmetic $(\alpha=1)$, geometric $(\alpha=0)$, and harmonic $(\alpha=-1)$ mean. Second, the sums $\sum\left|x-x_{j}\right|^{\alpha}$ can be considered 
as Riesz potentials (see [3]) of a discrete charge distribution with an atom of unit weight at each point $x_{j}$. The logarithmic $(\alpha=0)$ and the Newtonian $(\alpha=2-d)$ potential are special Riesz potentials.

The problem we are going to discuss is a problem of irregularities of distribution. If we replace the discrete distribution $\omega_{N}$ in (1) by the continuous uniform distribution $N \cdot \sigma$ on $S^{d-1}$, the corresponding integrals vanish identically on $S^{d-1}$. The fact that uniform distribution can be approximated by an $N$ point distribution to a certain degree of accuracy only implies the existence of certain lower bounds for the $L^{1}$-norm

$$
\left\|U_{\alpha}\left(x, \omega_{N}\right)\right\|_{1}=\frac{1}{\sigma(S)} \int_{S}\left|U_{\alpha}\left(x, \omega_{N}\right)\right| d \sigma(x) .
$$

We prove

Theorem 1. For each $N \geq 1$ and each $\alpha \neq 2,4, \ldots, 1-d<\alpha<$ $\infty$, the following inequality holds:

$$
\left\|U_{\alpha}\left(x, \omega_{N}\right)\right\|_{1} \geq c(d, \alpha) \cdot N^{-\alpha /(d-1)} .
$$

Here $c(d, \alpha)$ is a positive constant depending on $d$ and $\alpha$ only.

It will be proved in a later paper [10] that the result of Theorem 1 is best possible apart from the value of the constant $c(d, \alpha)$. Note that inequality (2) is false for $\alpha=2,4, \ldots$. In these exceptional cases, one can construct a point set $\omega_{N}$ for each $N \geq N_{0}(d, \alpha)$, such that $U_{\alpha}\left(x, \omega_{N}\right) \equiv 0$ on $S^{d-1}$. In the classical harmonic case $\alpha=2-d$, Theorem 1 is already contained in a paper by P. Sjögren [6]. After suitable choice of the parameters involved, his Theorem 1 implies that

$$
-\min _{x \in S} U_{2-d}\left(x, \omega_{N}\right) \geq c(d) \cdot N^{(d-2) /(d-1)},
$$

but his proof also applies to the case of the $L^{1}$-norm without any change. For $d=3$ and $\alpha=-1$, our result has the following physical interpretation: Suppose we place $N$ electrons (each of unit charge) on the surface $S^{2}$. The function $U_{-1}\left(x, \omega_{N}\right)$ measures the difference between the actual potential of $\omega_{N}$ at the point $x$, and its mean value which is equal to $N$. By Theorem 1 , there exist points $x \in S$ at which the actual potential is by at least $c \cdot N^{1 / 2}$ below the mean value. 
We also consider distance functionals $E_{\alpha}\left(\omega_{N}\right)$ which have the physical meaning of energy sums. Let

$$
\begin{aligned}
& E_{\alpha}\left(\omega_{N}\right)=\sum_{j, k}\left(\left|x_{j}-x_{k}\right|^{\alpha}-m(\alpha, d)\right) \quad \text { for } 0<\alpha<2, \\
& E_{0}\left(\omega_{N}\right)=\sum_{j \neq k}\left(\log \left|x_{j}-x_{k}\right|-m(0, d)\right), \quad \text { and } \\
& E_{\alpha}\left(\omega_{N}\right)=\sum_{j \neq k}\left(\left|x_{j}-x_{k}\right|^{\alpha}-m(\alpha, d)\right) \quad \text { for } 1-d<\alpha<0 .
\end{aligned}
$$

Note that when dealing with energy sums, we restrict ourselves to values of $\alpha$ satisfying $1-d<\alpha<2$.

If we replace the energy sums in (3) by the corresponding energy integrals with respect to uniform distribution $N \cdot \sigma$, we obtain the value zero. The fact that we approximate uniform distribution by a discrete distribution again gives rise to certain lower bounds for $E_{\alpha}\left(\omega_{N}\right)$. We prove

THEOREM 2. For each $N \geq 2$, the following energy inequalities hold:
(a) $E_{\alpha}\left(\omega_{N}\right) \leq-c(\alpha, d) \cdot N^{1-\alpha /(d-1)}$
$(0<\alpha<2)$,
(b) $E_{\alpha}\left(\omega_{N}\right) \geq-c(\alpha, d) \cdot N^{1-\alpha /(d-1)}$
$(1-d<\alpha<3-d)$,
(c) $E_{\alpha}\left(\omega_{N}\right) \geq-c(\alpha, d) \cdot N^{1-\alpha /(2-\alpha)}$
$(3-d \leq \alpha<0, d \geq 4)$,
(d) $E_{0}\left(\omega_{N}\right) \leq \frac{N}{2} \log N+O(N)$.

Let us make a few remarks. Theorem 2 is probably not best possible in the case (c), and in case (d) for $d \geq 4$. For $d=3$, the logarithmic case has already been handled in the author's paper [9].

The sum $E_{1}\left(\omega_{N}\right)$ was studied by K. B. Stolarsky ([7], [8]). He discovered a beautiful identity between the sum $E_{1}\left(\omega_{N}\right)$, and the $L^{2}$-norm of a function that measures discrepancy of the point set $\omega_{N}$ with respect to spherical caps on $S^{d-1}$. Using W. M. Schmidt's lower bounds for the discrepancy of an $N$ point set on $S^{d-1}$ with respect to spherical caps (see [5]), Stolarsky was able to obtain nontrivial bounds for $E_{1}\left(\omega_{N}\right)$ in dimension $d \geq 5$. J. Beck [1], using his method of Fourier transforms, finally proved the (best possible) estimate

$$
E_{1}\left(\omega_{N}\right) \leq-c(d) \cdot N^{(d-2) /(d-1)} .
$$

The method we shall use in order to prove Theorem 2 is independent of Beck's method. 
For $d=3$ and $\alpha=-1$, Theorem 2 contains the following result of physical interest. The energy $\sum_{j \neq k}\left|x_{j}-x_{k}\right|^{-1}$ of a distribution of $N$ electrons on $S^{2}$ satisfies the inequality

$$
\sum_{j \neq k}\left|x_{j}-x_{k}\right|^{-1} \geq N^{2}-c \cdot N^{3 / 2}
$$

For some basic facts on potential theory, we refer to the beautiful paper [4] by Polya and Szegö, and to Landkof's book [3]. The theory of spherical harmonics on $S^{d-1}$ is treated f.e. in [2].

2. Proof of Theorem 1. The proof of Theorem 1 is based on the construction of appropriate test functions $T(x)$ on $S^{d-1}$, and the use of the inequality

(4) $\left\|U_{\alpha}\left(x, \omega_{N}\right)\right\|_{1} \geq \frac{1}{\sigma(S)}\left|\int_{S} U_{\alpha}\left(x, \omega_{N}\right) T(x) d \sigma(x)\right| / \sup _{x \in S}|T(x)|$.

Step 1 . We introduce spherical coordinates $\theta=\left(\theta_{1}, \theta_{2}, \ldots, \theta_{d-2}\right)$ $\left(0 \leq \theta_{\rho} \leq \pi\right)$ and $\phi(0 \leq \phi<2 \pi)$ on $S^{d-1}$. Let $\Delta$ be the spherical Laplace operator on $S^{d-1}$, and consider the differential equation

$$
\begin{array}{r}
\Delta^{l} h_{l}\left(\cos \theta_{1}\right)=\left(\sin ^{2-d} \theta_{1} \frac{d}{d \theta_{1}}\left(\sin ^{d-2} \theta_{1} \cdot \frac{d}{d \theta_{1}}\right)\right)^{l} h_{l}\left(\cos \theta_{1}\right)=1, \\
l=1,2, \ldots .
\end{array}
$$

This equation has a solution on the interval $(0, \pi]$, which behaves like $\left(\sin \left(\theta_{1} / 2\right)\right)^{l-d+2}$ near the point $\theta_{1}=0$ for $l-d+2 \neq 0,2,4, \ldots$, and like $\left(\sin \left(\theta_{1} / 2\right)\right)^{l-d+2} \cdot \log \sin \left(\theta_{1} / 2\right)$ in the remaining cases. The expansion of $h_{l}$ into ultraspherical polynomials $P_{n}^{(\lambda)}\left(\cos \theta_{1}\right), \lambda=$ $\frac{d}{2}-1$, is given by

$$
h_{l}\left(\cos \theta_{1}\right) \sim c(\lambda, l) \cdot \sum_{n=1}^{\infty} \frac{n+\lambda}{(n(n+2 \lambda))^{l}} P_{n}^{(\lambda)}\left(\cos \theta_{1}\right) .
$$

The expansion (5), although not necessarily convergent, is known to be Poisson summable, which means

$$
h_{l}\left(\cos \theta_{1}\right)=\lim _{r \rightarrow 1} c(\lambda, l) \sum_{n=1}^{\infty} \frac{n+\lambda}{(n(n+2 \lambda))^{l}} r^{n} P_{n}^{(\lambda)}\left(\cos \theta_{1}\right)
$$

for $0<\theta_{1} \leq \pi$.

For the given point set $\omega_{N}$, consider the function

$$
H_{l}\left(x, \omega_{N}\right)=\sum_{j=1}^{N} h_{l}\left(\cos \gamma_{j}(x)\right) \text {, }
$$


where $2 \sin \left(\frac{1}{2} \gamma_{j}(x)\right)=\left|x-x_{j}\right|$ and $x \in S^{d-1}$. For the function $H_{l}$, the inequality $\|\left. H_{l}\left(x, \omega_{N}\right)\right|_{1} \gg N^{1-2 l /(d-1)}$ is easily proved in the following way.

Consider the subdomain $D \subset S^{d-1}$ determined by the relations $0 \leq \frac{\pi}{2}-\theta_{\rho} \leq \frac{\pi}{6} \quad(\rho=1,2, \ldots, d-2)$, and $0 \leq \phi \leq \frac{\pi}{6}$. Let $r=r(N)$ be the integer satisfying

$$
2 N \leq 2^{(d-1) r}<2^{d} \cdot N .
$$

We partition $D$ into "cubes" $B_{\mu}=B_{\mu_{1} \mu_{2} \cdots \mu_{d-1}}\left(1 \leq \mu_{\rho} \leq 2^{r}\right)$, where $B_{\mu}$ is determined by the inequalities

$$
\begin{gathered}
\left(\mu_{\rho}-1\right) \cdot \frac{\pi}{6} \cdot 2^{-r} \leq \theta_{\rho} \leq \mu_{\rho} \cdot \frac{\pi}{6} \cdot 2^{-r} \quad(\rho=1,2, \ldots, d-2) \quad \text { and } \\
\left(\mu_{d-1}-1\right) \cdot \frac{\pi}{6} \cdot 2^{-r} \leq \phi \leq \mu_{d-1} \cdot \frac{\pi}{6} \cdot 2^{-r} .
\end{gathered}
$$

The set of subdomains $B_{\mu}$ containing none of the points $x_{j}$ in their interior will be denoted by $\Lambda$. By the choice of $r$, we have

$$
\sum_{\Lambda} \sigma\left(B_{\mu}\right) \gg 1
$$

For $x=(\theta, \phi) \in B_{\mu} \in \Lambda$, let

$$
\tau_{\mu}(x)=4^{-l r} \prod_{\rho=1}^{d-2} \sin ^{2 l} 6 \cdot 2^{r} \theta_{\rho} \cdot \sin ^{2 l} 6 \cdot 2^{r} \phi .
$$

Define a test function $T(x)$ on $S^{d-1}$ by putting

$$
T(\theta, \phi)=\Delta^{l} \tau_{\mu}(\theta, \phi)
$$

for $(\theta, \phi) \in B_{\mu} \in \Lambda$, and $T(\theta, \phi)=0$ elsewhere. Note that $\sup _{x \in S}|T(x)| \ll 1$ holds. Multiplying $H_{l}\left(x, \omega_{N}\right)$ by $T(x)$, and integrating over $S^{d-1}$, we obtain, using Green's second formula:

(6) $\left|\int_{S} H_{l}\left(x, \omega_{N}\right) T(x) d \sigma(x)\right|=\left|\sum_{\Lambda} \int_{B_{\mu}} H_{l}\left(x, \omega_{N}\right) \Delta^{l} \tau_{\mu}(x) d \sigma(x)\right|$

$$
\begin{aligned}
& =\left|\sum_{\Lambda} \int_{B_{\mu}} \Delta^{l} H_{l}\left(x, \omega_{N}\right) \cdot \tau_{\mu}(x) \cdot d \sigma(x)\right| \\
& \gg N \cdot \sum_{\Lambda} \int_{B_{\mu}} \tau_{\mu}(x) d \sigma(x) \gg N \cdot 4^{-l r} \gg N \cdot N^{-2 l /(d-1)} .
\end{aligned}
$$

Here we use the fact that the normal derivatives of $\Delta^{m} \tau_{\mu}(x) \quad(m=$ $0,1, \ldots, l-1)$ with respect to the boundary of $B_{\mu}$ vanish. From 
relations (4) and (6), using $\sup _{x \in S}|T(x)| \ll 1$, the inequality $\left\|H_{l}\left(x, \omega_{N}\right)\right\|_{1} \gg N \cdot N^{-2 l /(d-1)}$ follows.

Step 2. We begin with the case $1-d<\alpha<3-d$. Consider the kernel $k_{\alpha}\left(\cos \theta_{1}\right)=\left|2 \sin \left(\theta_{1} / 2\right)\right|^{\alpha}$, which generates the distance function $|x-y|^{\alpha}$. We are looking for an inverse kernel $k_{\alpha}^{-1}\left(\cos \theta_{1}\right)$ such that the convolution equation

$$
k_{\alpha}^{-1} * k_{\alpha}=h_{1}
$$

holds on $S^{d-1}$.

We have the expansion

$$
k_{\alpha}\left(\cos \theta_{1}\right) \sim \sum_{n=0}^{\infty} a_{n} \cdot P_{n}^{(\lambda)}\left(\cos \theta_{1}\right)
$$

where

$$
\begin{aligned}
a_{n} & =c(\lambda, \alpha) \cdot \frac{(n+\lambda) \cdot \Gamma(n-\alpha / 2)}{\Gamma(n+2 \lambda+1+\alpha / 2)} \text { and } \\
c(\lambda, \alpha) & =2^{1+\alpha} \cdot \frac{\Gamma(2 \lambda) \cdot \Gamma(\alpha / 2+\lambda+1 / 2)}{\Gamma(\lambda+1 / 2) \cdot \Gamma(-\alpha / 2)} .
\end{aligned}
$$

Note that the expansion (8) holds for any value of $\alpha$ satisfying $1-d<$ $\alpha$ and $\alpha \neq 0,2, \ldots$. If we omit the factor $\Gamma\left(-\frac{\alpha}{2}\right)$ in the denominator $c(\lambda, \alpha)$, we obtain a kernel of the type $\left|\sin \left(\theta_{1} / 2\right)\right|^{\alpha} \log \sin \left(\theta_{1} / 2\right)$ for these exceptional values of $\alpha$. It is in this sense that we shall use the notation $k_{\alpha}\left(\cos \theta_{1}\right)$ for all $\alpha>1-d$.

Proceeding quite formally, and using (5), we obtain a solution of (7) in the form

$$
k_{\alpha}^{-1}\left(\cos \theta_{1}\right) \sim \sum_{n=1}^{\infty} b_{n} \cdot P_{n}^{(\lambda)}\left(\cos \theta_{1}\right),
$$

where

$$
b_{n}=c_{1}(\lambda, \alpha) \cdot \frac{(n+\lambda)^{2}}{n(n+2 \lambda)} \cdot \frac{\Gamma(n+2 \lambda+1+(\alpha / 2))}{(n+\lambda) \cdot \Gamma(n-(\alpha / 2))} .
$$

Using Stirling's formula, and subtracting successively appropriate multiples of (8) (with $\alpha$ replaced by $4-2 d-\alpha, 5-2 d-\alpha, \ldots$ ) from (9), we obtain a representation

$$
\begin{array}{r}
k_{\alpha}^{-1}=d_{1} \cdot k_{4-2 d-\alpha}+d_{2} \cdot k_{5-2 d-\alpha}+\cdots+d_{s} \cdot k_{s+3-2 d-\alpha}+R_{s} \\
(d-1 \neq 0),
\end{array}
$$

where $\Delta R_{s}$ is bounded and continuous for $s=d+1$. 
A rigorous proof of (10) is obtained in the following way. Let

$$
\pi_{r}\left(\cos \theta_{1}\right)=\sum_{n=0}^{\infty}(n+\lambda) r^{n} \cdot P_{n}^{(\lambda)}\left(\cos \theta_{1}\right) \quad(0<r<1)
$$

be the Poisson kernel on $S^{d-1}$. Note that $k_{\alpha}^{-1}$ and $h_{1}$ are integrable over $S^{d-1}$, and hence that $\kappa=k_{\alpha}^{-1} * \pi_{r}$ solves the equation

$$
\kappa * k_{\alpha}=h_{1} * \pi_{r} \text {. }
$$

Letting $r \rightarrow 1$, we obtain the desired result.

From (10), we further get the estimates

$$
\begin{aligned}
& \left|k_{\alpha}^{-1}(|x-y|)\right| \ll|x-y|^{4-2 d-\alpha} \text { and } \\
& \left|\Delta k_{\alpha}^{-1}(|x-y|)\right| \ll|x-y|^{2-2 d-\alpha} .
\end{aligned}
$$

Step 3. We use $T * k_{\alpha}^{-1}$ as a test function for $U_{\alpha}\left(x, \omega_{N}\right)$, where $T$ is the test function introduced in step 1 . In view of the relation

$$
\begin{array}{r}
\int_{S} U_{\alpha}\left(x, \omega_{N}\right) \cdot\left(T * k_{\alpha}^{-1}\right)(x) d \sigma(x) \\
=\int_{S}\left(U_{\alpha} * k_{\alpha}^{-1}\right)(x) \cdot T(x) d \sigma(x) \\
=\int_{S} H_{1}\left(x, \omega_{N}\right) \cdot T(x) d \sigma(x),
\end{array}
$$

it is sufficient to estimate $\sup _{x \in S}\left|\left(T * k_{\alpha}^{-1}\right)(x)\right|$.

For fixed $x \in S^{d-1}$, let $\Lambda^{\prime}=\Lambda_{x}^{\prime}$ be the set of subdomains $B_{\mu} \in \Lambda$ that contain some point $y$ such that $|x-y|<N^{-1 /(d-1)}$ holds. Let $\Lambda^{\prime \prime}=\Lambda \backslash \Lambda^{\prime}$ be the set of remaining $B_{\mu}$ 's. We have

$$
\begin{aligned}
\left|\left(T * k_{\alpha}^{-1}\right)(x)\right| \ll & \left|\sum_{\Lambda^{\prime}} \int_{B_{\mu}} \Delta \tau_{\mu}(y) \cdot k_{\alpha}^{-1}(|x-y|) d \sigma(y)\right| \\
& +\left|\sum_{\Lambda^{\prime \prime}} \int_{B_{\mu}} \tau_{\mu}(y) \cdot \Delta k_{\alpha}^{-1}(|x-y|) d \sigma(y)\right| \\
\ll & \sum_{\Lambda^{\prime}} \int_{B_{\mu}}|x-y|^{4-2 d-\alpha} d \sigma(y) \\
& +\sum_{\Lambda^{\prime \prime}} \int_{B_{\mu}}|x-y|^{2-2 d-\alpha} d \sigma(y) \\
\ll & N^{(d+\alpha-3) /(d-1)} .
\end{aligned}
$$

From (6), (12), and (13), the assertion follows. 
In order to obtain the assertion of Theorem 1 in the case $2 l-1-d<$ $\alpha<2 l+1-d(l=2,3, \ldots ; \alpha \neq 2,4, \ldots)$, we proceed in a similar way, solving the equation $k_{\alpha}^{-1} * k_{\alpha}=h_{l}$, and noting that

$$
\sup _{x \in S}\left|\left(T * k_{\alpha}^{-1}\right)(x)\right| \ll N \cdot N^{(\alpha-2 l) /(d-1)} .
$$

This argument also works for $\alpha=2 l-1-d, \alpha \neq 2,4, \ldots$, whereas in the case $\alpha=2,4, \ldots$ the convolution equation which corresponds to (7) has no solution. However, if we define $U_{\alpha}\left(x, \omega_{N}\right)$ by

$$
U_{\alpha}\left(x, \omega_{N}\right)=\sum_{j=1}^{N}\left|x-x_{j}\right|^{\alpha} \log \left|x-x_{j}\right|-N \cdot m^{\prime}(\alpha, d)
$$

for $\alpha=2,4, \ldots$, the assertion of Theorem 1 would also remain true in the exceptional cases.

This finishes our proof of Theorem 1.

3. Bounds for energy sums. In proving Theorem 2, we shall distinguish three cases.

The case $0<\alpha<2$. By formula (8), all the coefficients $a_{n}=a_{n}(\alpha)$ $(n \geq 1)$ in the expansion of $k_{\alpha}\left(\cos \theta_{1}\right)$ are negative. The addition formula for spherical harmonics (see [2], §11.4.) implies the following identity:

$$
E_{\alpha}\left(\omega_{N}\right)=-c(\alpha, d) \int_{S}\left(\sum_{j=1}^{N} \delta_{\alpha}\left(\left|x-x_{j}\right|\right)\right)^{2} d \sigma(x)
$$

Here $c(\alpha, d)$ is a positive constant, and $\delta_{\alpha}\left(\left|x-x_{j}\right|\right)$ is a new distance function, generated by the kernel

$$
\delta_{\alpha}\left(\cos \theta_{1}\right) \sim \sum_{n=1}^{\infty}\left(-(n+\lambda) \cdot a_{n}(\alpha)\right)^{1 / 2} \cdot P_{n}^{(\lambda)}\left(\cos \theta_{1}\right) .
$$

In view of the expansion (8), the kernel $\delta_{\alpha}\left(\cos \theta_{1}\right)$ is of the type $\delta_{\alpha}(|x-y|) \sim|x-y|^{(1+\alpha-d) / 2}$. Now choose the integer $l \geq 1$ such that $2 l-1-d \leq(1+\alpha-d) / 2<2 l+1-d$. Consider again the convolution equation

$$
\delta_{\alpha}^{-1} * \delta_{\alpha}=h_{l}
$$

Proceeding as in the proof of Theorem 1, we find that the inverse $\delta_{\alpha}^{-1}$ has a representation of the following form:

$$
\delta_{\alpha}^{-1}=\sum_{m=1}^{s} d_{m} \cdot k_{2 l+m+1-2 d-\beta}+R_{s} \quad\left(d_{1} \neq 0\right) .
$$


Here $\beta=(1+\alpha-d) / 2$, and if we choose $s$ large enough, $\Delta^{l} R_{s}$ will be bounded on $S$. From now on the proof is the same as in step 3 of the proof of Theorem 1, yielding

$$
\left\|\sum_{j=1}^{N} \delta_{\alpha}\left(\left|x-x_{j}\right|\right)\right\|_{1} \gg N^{(d-\alpha-1) / 2(d-1)} .
$$

From this and the Cauchy-Schwarz inequality, the inequality

$$
E_{\alpha}\left(\omega_{N}\right) \leq-c(\alpha, d) \cdot N^{1-\alpha /(d-1)}
$$

follows immediately.

The case $1-d<\alpha<3-d$. In the case of an unbounded kernel $k_{\alpha}$, we have to proceed in a different way. Together with the kernel $k_{\alpha}(\theta)=\left(2-2 \cos \theta_{1}\right)^{\alpha / 2}$ consider the more general kernel

$$
d_{r}\left(\cos \theta_{1}\right)=\left(r+\frac{1}{r}-2 \cos \theta_{1}\right)^{\alpha / 2} \quad(0<r \leq 1) .
$$

Let $m_{r}$ be the mean value of $d_{r}\left(\cos \theta_{1}\right)$ over $S^{d-1}$, and let $d_{r}(|x-y|)$ be the distance function generated by $d_{r}\left(\cos \theta_{1}\right)$ on $S^{d-1}$. We have

$$
\begin{aligned}
E_{\alpha}\left(\omega_{N}\right)= & \sum_{j \neq k}\left(d_{1}\left(\left|x_{j}-x_{k}\right|\right)-m_{1}\right) \\
= & \sum_{j, k}\left(d_{r}\left(\left|x_{j}-x_{k}\right|\right)-m_{r}\right)-N \cdot d_{r}(0) \\
& -N^{2} \cdot\left(m_{1}-m_{r}\right)+N \cdot m_{1} \\
& +\sum_{j \neq k}\left(d_{1}\left(\left|x_{j}-x_{k}\right|\right)-d_{r}\left(\left|x_{j}-x_{k}\right|\right)\right) .
\end{aligned}
$$

First of all note that $d_{r}(|x-y|) \leq d_{1}(|x-y|)$, and that

$$
\sum_{j, k}\left(d_{r}\left(\left|x_{j}-x_{k}\right|\right)-m_{r}\right) \geq 0
$$

as all the coefficients of $d_{r}\left(\cos \theta_{1}\right)-m_{r}$ in the ultraspherical expansion are nonnegative. (This may be proved in the same way as Hilfssatz 6 in [4], using the Rodrigues formula for ultraspherical polynomials.)

Hence

$$
E_{\alpha}\left(\omega_{N}\right) \geq-N \cdot d_{r}(0)-N^{2} \cdot\left(m_{1}-m_{r}\right) .
$$

Now choose $r=1-N^{-1 /(d-1)}$. We have $d_{r}(0) \ll N^{-\alpha /(d-1)}$ and $m_{1}-m_{r} \ll N^{-1} \cdot N^{-\alpha /(d-1)}$. Inserting these estimates in (14) yields the desired result. 
The case $3-d \leq \alpha \leq 0, d \geq 4$. Unfortunately, the preceding method does not seem to give the best result in the case $3-d<\alpha<0$. Putting $r=1-\varepsilon$, we obtain $d_{r}(0) \ll \varepsilon^{\alpha}$ and $m_{1}-m_{r} \ll \varepsilon^{2}$ (instead of $\varepsilon^{d-1+\alpha}$ as above). Choosing $\varepsilon=N^{1 /(2-\alpha)}$, assertion (c) of Theorem 2 follows.

In the logarithmic case, the same procedure yields

$$
E_{0}\left(\omega_{N}\right) \leq \frac{N}{2} \log N+O(N),
$$

which is best possible in dimension 3 (see [9]), but probably not in higher dimensions. This finishes our proof of Theorem 2.

\section{REFERENCES}

[1] J. Beck, On the sum of distances between $N$ points on a sphere, Mathematika, 31 (1984), 33-41.

[2] E. Erdelyi, W. Magnus, F. Oberhettinger and F. G. Tricomi, Higher Transcendental Functions, Volume 2. McGraw-Hill (1953).

[3] N. S. Landkof, Foundations of Modern Potential Theory, Springer-Verlag (1972).

[4] G. Polya and G. Szegö, On the transfinite diameter (capacity constant) of subsets in the plane and in space (german), J. für Reine und Angew. Math., 165 (1931), 4-49.

[5] W. M. Schmidt, Irregularities of distribution VII, Acta Arith., 21 (1972), 45-50.

[6] P. Sjögren, Estimates of mass distributions from their potentials and energies, Arkiv för mat., 10 (1972), 59-77.

[7] K. B. Stolarsky, Sums of distances between points of a sphere, Proc. Amer. Math. Soc., 35 (1972), 547-549.

[8] _- Sums of distances between points of a sphere II, Proc. Amer. Math. Soc., 41 (1973), 575-582.

[9] G. Wagner, On the product of distances to a point set on a sphere, J. Australian Math. Soc., (Series A) 47 (1989), 466-482.

[10] _ On a new method for constructing good point sets on spheres, to appear.

Received September 23, 1988.

Mathematisches Institut A

PFAFFenWALdRING 57

D-7000 StutTGART 80 (FRG) 


\section{PACIFIC JOURNAL OF MATHEMATICS EDITORS}

\author{
V. S. VARADARAJAN \\ (Managing Editor) \\ University of California \\ Los Angeles, CA 90024-1555-05 \\ Herbert Clemens \\ University of Utah \\ Salt Lake City, UT 84112 \\ THOMAS ENRIGHT \\ University of California, San Diego \\ La Jolla, CA 92093
}

R. FINN

Stanford University

Stanford, CA 94305

Hermann Flaschka

University of Arizona

Tucson, AZ 85721

VAUGHAN F. R. Jones

University of California

Berkeley, CA 94720

Steven Kerckhoff

Stanford University

Stanford, CA 94305

\author{
C. C. MOORE \\ University of California \\ Berkeley, CA 94720
}

MARTIN SCHARLEMANN

University of California

Santa Barbara, CA 93106

HAROLd STARK

University of California, San Diego

La Jolla, CA 92093

\section{ASSOCIATE EDITORS}
R. ARENS
E. F. BECKENBACH
B. H. NeUmanN
F. WoLF
(1904-1989)
K. YosHIDA
(1906-1982)

\section{SUPPORTING INSTITUTIONS}

UNIVERSITY OF ARIZONA
UNIVERSITY OF BRITISH COLUMBIA
CALIFORNIA INSTITUTE OF TECHNOLOGY
UNIVERSITY OF CALIFORNIA
MONTANA STATE UNIVERSITY
UNIVERSITY OF NEVADA, RENO
NEW MEXICO STATE UNIVERSITY
OREGON STATE UNIVERSITY

UNIVERSITY OF ARIZONA

CAIVERSITY OF BRITISH COLUN UNIVERSITY OF CALIFORNIA

MONTANA STATE UNIVERSITY OREGON STATE UNIVERSITY

\author{
UNIVERSITY OF OREGON \\ UNIVERSITY OF SOUTHERN CALIFORNIA \\ STANFORD UNIVERSITY \\ UNIVERSITY OF HAWAII \\ UNIVERSITY OF TOKYO \\ UNIVERSITY OF UTAH \\ WASHINGTON STATE UNIVERSITY \\ UNIVERSITY OF WASHINGTON
}

The Supporting Institutions listed above contribute to the cost of publication of this Journal, but they are not owners or publishers and have no responsibility for its content or policies.

Mathematical papers intended for publication in the Pacific Journal of Mathematics should be in typed form or offset-reproduced (not dittoed), double spaced with large margins. Please do not use built up fractions in the text of the manuscript. However, you may use them in the displayed equations. Underline Greek letters in red, German in green, and script in blue. The first paragraph must be capable of being used separately as a synopsis of the entire paper. In particular it should contain no bibliographic references. Please propose a heading for the odd numbered pages of less than 35 characters. Manuscripts, in triplicate, may be sent to any one of the editors. Please classify according to the 1980 Mathematics Subject Classification (1985 Revision) scheme which can be found in the December index volumes of Mathematical Reviews. Supply name and address of author to whom proofs should be sent. All other communications should be addressed to the managing editor, or Elaine Barth, University of California, Los Angeles, California 90024-1555-05.

There are page-charges associated with articles appearing in the Pacific Journal of Mathematics. These charges are expected to be paid by the author's University, Government Agency or Company. If the author or authors do not have access to such Institutional support these charges are waived. Single authors will receive 50 free reprints; joint authors will receive a total of 100 free reprints. Additional copies may be obtained at cost in multiples of 50 .

The Pacific Journal of Mathematics (ISSN 0030-8730) is published monthly. Regular subscription rate: $\$ 190.00$ a year (12 issues). Special rate: $\$ 95.00$ a year to individual members of supporting institutions.

Subscriptions, orders for numbers issued in the last three calendar years, and changes of address should be sent to Pacific Journal of Mathematics, P.O. Box 969, Carmel Valley, CA 93924, U.S.A. Old back numbers obtainable from Kraus Periodicals Co., Route 100, Millwood, NY 10546.

The Pacific Journal of Mathematics at P.O. Box 969, Carmel Valley, CA 93924 (ISSN 0030-8730) is published monthly. Second-class postage paid at Carmel Valley, California 93924, and additional mailing offices. Postmaster: send address changes to Pacific Journal of Mathematics, P.O. Box 969, Carmel Valley, CA 93924.

\section{PUBLISHED BY PACIFIC JOURNAL OF MATHEMATICS, A NON-PROFIT CORPORATION}




\section{Pacific Journal of Mathematics}

Vol. 144, No. $2 \quad$ June, 1990

George E. Andrews and David M. Jackson, An algebraically derived $q$-analogue of a character sum associated with a class of semiregular

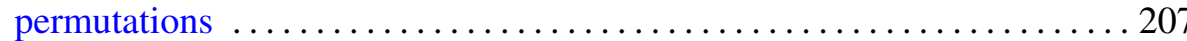

Fabio Bardelli and Andrea Del Centina, The moduli space of genus four double covers of elliptic curves is rational $\ldots \ldots \ldots \ldots \ldots \ldots \ldots \ldots 219$

Young Do Chai, An estimate of the volume of a compact set in terms of its



Salvador Comalada, Elliptic curves with trivial conductor over quadratic fields

Kahar El-Hussein, Résolubilité semi-globale des opérateurs différentiels invariants sur les groupes de déplacements $\ldots \ldots \ldots \ldots \ldots \ldots \ldots \ldots . \ldots 259$

David M. Goldschmidt, Classical link invariants and the Burau representation

Liliana Janicka, Radon-Nikodým problem for the variation of a vector measure

Wacław Marzantowicz, An almost classification of compact Lie groups with Borsuk-Ulam properties ............................ 299

Akira Ohbuchi, On the projective normality of some varieties of degree 5 . . 313 Ken'ichi Ohshika, Minimal measured laminations in geometric

3-manifolds

Hal Leslie Smith, A discrete Lyapunov function for a class of linear differential equations

John Samuel Spielberg, Diagonal states on $O_{2} \ldots \ldots \ldots \ldots \ldots \ldots \ldots \ldots$

Thomas Vogel, A note on the sessile drop

Gerold Wagner, On means of distances on the surface of a sphere (lower bounds) 